\title{
Laser Confocal Scanning Microscopy and Transmission Electron Microscopy to Visualize the Site of Callose Fiber Elongation on a Single Conifer Protoplast Selected With a Micromanipulator
}

\author{
Tomoya Oyanagi ${ }^{1}$, Asami Kurita ${ }^{1}$, Takeshi Fukumoto ${ }^{2}$, Noriko Hayashi ${ }^{3} \&$ Hamako Sasamoto ${ }^{1,4}$ \\ ${ }^{1}$ Graduate School of Environment and Information Sciences, Yokohama National University, Japan \\ ${ }^{2}$ Faculty of Agriculture, Department of Life Sciences, Kagawa University, Japan \\ ${ }^{3}$ Forestry and Forest Products Research Institute, Japan \\ ${ }^{4}$ Faculty of Environment and Information Sciences, Yokohama National University, Japan \\ Correspondence: Hamako Sasamoto, Faculty of Environment and information Sciences, Yokohama National \\ University, Yokohama 240-8501, Japan. Tel: 81-45-339-4414. E-mail: sasamoto@ynu.ac.jp
}

Received: February 12, 2013 Accepted: March 12, 2014 Online Published: March 24, 2014

doi:10.5539/jps.v3n2p23

URL: http://dx.doi.org/10.5539/jps.v3n2p23

\begin{abstract}
We have developed a new method to observe the site of big and long spiral callose fiber elongation from a protoplast in liquid culture medium. Protoplasts of embryogenic cells of a conifer, Larix leptolepis, were cultured in the $\mathrm{NH}_{4} \mathrm{NO}_{3}$-free Murashige and Skoog's medium containing $50 \mathrm{mM} \mathrm{MgCl} 2$ and $6 \%$ sucrose in a well in a 96or 24-well culture plates. The protoplasts with elongated callose fibers were selected before fixation with glutaraldehyde, by picking up using a micromanipulator after electric treatment (DC $3 \mathrm{kV} / \mathrm{cm}$ ) in the medium containing Alexafluor 488 phalloidin. Under laser confocal scanning microscopy (LCSM), two sites in the cell were stained clearly. One site was the nucleus and the other was the plasma membrane from which fibers elongated. Single cell transmission electron microscopy (TEM) was developed for observation of the microstructure at the site of fiber elongation on a single protoplast. A single protoplast which had a fiber was selected using a micromanipulator and transferred to an agarose bead at a low gelling temperature. The cells were fixed with cold glutaraldehyde and processed for TEM analysis. Elongated thin fibrils with vesicle-like structures could be observed by TEM.
\end{abstract}

Keywords: $\beta$-1,3-glucan, conifer, fiber structure, protoplast culture, ultra-structure

\section{Introduction}

We have reported novel long and large spiral callose fibers developed in protoplast cultures of several plant species, i.e., a conifer Larix leptolepis, a broad-leaved tree, Betula platyphylla, and herbaceous plants, Nicotiana tabacum and Vinca rosea, by treatment with high concentrations of $\mathrm{MgCl}_{2}$ or $\mathrm{CaCl}_{2}$ in a liquid medium (Sasamoto et al., 2003; Fukumoto et al., 2005; Fukumoto, 2006). Fibers were stained with a fluorescent dye, Aniline Blue. Callose fibers of L. leptolepis were confirmed by specific enzymatic degradation of $\beta$-1,3-glucan with laminarinase treatment (Fukumoto et al., 2005). Substructures of the callose fiber, i.e., fibrils and sub-fibrils, which are very large compared to the cellulose ( $\beta$-1,4-glucan) microfibrils, were observed using transmission electron microscopy (TEM) (Sasamoto et al., 2003), laser confocal scanning microscopy (LCSM) and atomic force microscopy (Fukumoto et al., 2005). Recently, some callose fibers were also found in protoplast cultures of several tree mangrove species, without high salt concentrations in the medium, and their callose component was confirmed by an enzymatic method (Kurita et al., 2008).

We reported a specific site at the surface of $L$. Leptolepis protoplasts where a fiber appeared. The present study was conducted to visualize the intracellular structure associated with fiber formation using a protoplast culture of L. Leptolepis (Sasamoto et al., 2003).

Phalloidin, which interacts with actin protein, is used for visualization of the cytoskeleton for cellulose microfibril deposition (Wightman \& Turner, 2008). However, phalloidin is difficult to permeate through the cell membrane. Here, we report a novel method using LCSM after electric treatment that promoted the entry of phalloidin into protoplasts for observing the intracellular site of callose fiber formation. Furthermore, by using 
micromanipulation techniques developed for selection of fused protoplasts (Sasamoto et al., 2000), we improved the single cell TEM technique (Faure et al., 1992) for observing the site of (sub)-fibrils formation.

\section{Method}

\subsection{Cell Culture}

Embryogenic suspension cells of L. leptolepis (Ogita et al., 1999) were sub-cultured every three weeks in liquid mCD medium containing $7 \mu \mathrm{M}$ of 2,4-dichlorophenoxyacetic acid (2,4-D), $3 \mu \mathrm{M}$ of benzyladenine (BA), $3 \%$ sucrose and $2000 \mathrm{mg} / \mathrm{L}$ of glutamine (Fukumoto et al., 2005; Fukumoto, 2006). The $\mathrm{pH}$ was adjusted to 5.6 before sterilization at $121{ }^{\circ} \mathrm{C}$ for $20 \mathrm{~min}$. The cells were cultured at $25^{\circ} \mathrm{C}$ in $10-15 \mathrm{~mL}$ medium in a $100 \mathrm{~mL}$ glass culture flask (CUL-FK100, Iwaki) on a rotary shaker at $100 \mathrm{rpm}$ speed. Light condition was $16 \mathrm{hr}$ light photoperiod at $60 \mu \mathrm{mol} \mathrm{m} \mathrm{m}^{-2}$.

\subsection{Protoplast Isolation and Culture}

Protoplasts were isolated as previously described (Fukumoto et al., 2005) from 3 week-old suspension cells by a combination of $1 \%$ Cellulase RS (Yakult) and $0.05 \%$ Pectolyase Y-23 (Seishin) in $0.4 \mathrm{M}$ mannitol solution for 1-2 hrs. Protoplasts were purified by passing through a $42 \mu \mathrm{m}$ sized nylon mesh and washed by centrifugation at $100 \times \mathrm{g}$ for 4 min with mannitol solution 3 times. Protoplasts were cultured in liquid $\mathrm{NH}_{4} \mathrm{NO}_{3}$-free Murashige and Skoog's (MS, Murashige \& Skoog, 1962) basal medium containing $10 \mu \mathrm{M}$ each of 2,4-D and BA, 6\% sucrose and $50 \mathrm{mM} \mathrm{MgCl}_{2}$ in a 96 well (No. 3075, Falcon) or 24 well (No. 3047, Falcon) culture plate. Hundred $\mu \mathrm{L}$ or $1 \mathrm{~mL}$ of pure water was supplied to the space between wells for humid condition. Protoplast density was 2 $\times 10^{4} \mathrm{~mL}^{-1}$. Culture was maintained at $28{ }^{\circ} \mathrm{C}$ in a humid incubator $\left(\mathrm{CO}_{2}\right.$-incubator, Astec APC 30D/CL-30) without the supply of $\mathrm{CO}_{2}$.

\subsection{Aniline Blue Staining}

Aniline blue solution $(0.5 \%)$ in $20 \mathrm{mM}$ phosphate buffer ( $\mathrm{pH} 8.5$ ) was added to the culture medium to stain fibers at a final concentration of $0.05 \%$ (Sasamoto et al., 2003; Fukumoto et al., 2005). Fluorescence was observed under a fluorescence inverted microscope (Olympus IX71) equipped with a UV filter (U-MWU2).

\subsection{DAPI Staining With Fixation}

Fiber-forming protoplasts after culture were selected and transferred into the medium in a well of a 4-well plastic plate (multidish 4 well, Nunc; No. 3654, Falcon) by using a micromanipulator (Narishige-Nikon MM-188) attached to an inverted microscope (Olympus CK40 or IX71). Fixation with 1\% glutaraldehyde in the medium for protoplast culture was performed for $30 \mathrm{~min}$. Then the protoplasts were stained with 4',6-diamidino-2-phenylindole (DAPI) at a final concentration of $1 \mathrm{ng} \mathrm{mL} \mathrm{m}^{-1}$. UV Fluorescence was observed as described for Aniline Blue staining.

\subsection{Phalloidin Staining With Electric Treatment}

Fiber-forming protoplasts cultured for about one week were stained with Alexa Fluor 488 Phalloidin (A12379 Invitrogen, Molecular Probe) at a final concentration of $14 \mathrm{nM}$ by electric treatment. Electric treatment was performed using a slide glass electrode ( $2 \mathrm{~mm}$ gap CUY501G2L, NEPA GENE). A $330 \mu \mathrm{L}$ volume of cultured medium containing fiber-forming protoplasts and $30 \mu \mathrm{L}$ of phalloidin solution in $0.1 \mathrm{M}$ phosphate buffe ( $\mathrm{pH} 7.0)$ were placed between the slide electrodes. A direct current (DC) pulse of $1-4 \mathrm{kV} / \mathrm{cm}$ was applied for $99 \mu \mathrm{sec}$ using electro cell fusion generator (LF-101, NEPA GENE). After electric treatment, the liquid was transferred to a well in a 4-well plastic plate using a Pipetman before viability determination and LCSM. When cited in the text, protoplasts were stained with both DAPI and phalloidin.

\subsection{Viability of Protoplasts}

The viability of protoplasts was determined by staining with fluorescein diacetate (FDA) (Larkin, 1976) at a final concentration of $0.01 \%$. Protoplasts were counted after taking photographs using a digital camera system (NY1S, Micronet corp.) under a fluorescence inverted microscope (Olympus CK40).

\subsection{Micromanipulation}

Fiber-forming protoplasts were selected using micromanipulators (Narishige MM-89, Narishige-Nikon MM-188) with a microinjector (IM-188) under inverted microscopes (Olympus IX-71, CK-40). Micropipettes for picking up protoplasts were made from a glass capillary (10 $\mu \mathrm{L}$, Drummond) using a micropipette puller (PB-7, Narishige), and were bent with a micro-burner (Pen burner, Prince) as previously described (Ogita et al., 1999; Sasamoto et al., 2000). 


\subsection{LCSM}

After transferring the fiber-forming protoplasts stained with phalloidin to a glass base dish $(3 \mathrm{~cm} \varphi$, Iwaki) or glass bottom dish (Corning) using a micromanipulator, fluorescence was observed at $488 \mathrm{~nm}$ and $408 \mathrm{~nm}$ with a laser confocal scanning microscope (Nikon C1, Differential interference contrast (DIC) and fluorescence inverted microscope, Nikon).

\subsection{Single Cell TEM}

A single fiber-forming protoplast was picked up using a micromanipulator as described above, transferred to a droplet of microbeads of agarose (6-8 $\mu \mathrm{L}$, low gelling temperature type \#7, A4018, and ultra-low gelling temperature type \#9, A5030, Sigma) before solidification at room temperature in a well in a 4-well plastic plate. Agarose was dissolved in the medium for protoplast culture. The beads were fixed with cold $2 \%$ glutaraldehyde solution for $1 \mathrm{hr}$ at $4{ }^{\circ} \mathrm{C}$. They were washed with $0.1 \mathrm{M}$ phosphate buffer $(\mathrm{pH} 7.0)$, and transferred to a $1.5 \mathrm{~mL}$ microtube (MCT-150-C, Axygen) with a small spatula. They were treated with $1 \%$ osmium tetroxide at $4{ }^{\circ} \mathrm{C}$, washed with ultra pure water several times, successively dehydrated with ethanol and acetone series, and embedded in LRwhite resin. Ultrathin sections were put on a grid with thin formvar film, then stained with $1 \%$ uranyl acetate and $\mathrm{Pb}$-citrate and examined using a JEM-2000EX (JEOL) transmission electron microscope operated at $200 \mathrm{kV}$ (Hayashi et al., 1997a, b; Sasamoto et al., 2003).

\section{Results \& Discussion}

\subsection{Observation of Intracellular Structures for Fiber Formation}

\subsubsection{Observation With a Fluorescence Inverted Microscope}

After one to two weeks for culturing protoplasts from L. leptolepis, fiber formation was observed under an inverted fluorescence microscope. Fibers were stained with Aniline Blue (data not shown) as reported previously (Sasamoto et al., 2003; Fukumoto et al., 2005). Using DAPI without fixation of cells, the surface of protoplasts forming fibers was stained, but intracellular structures were difficult to be discerned (Figures 1a, b). As elongated fibers were all released after pre-fixation in glutaraldehyde solution in preliminary experiments, fiber-forming protoplasts (Figure 1c) were selected, picked up using a micromanipulator, transferred to another well on a 4-well plastic plate, and stained using a combination of glutaraldehyde and DAPI solutions. Using this approach, DAPI penetrated the cell membrane, and the four and two circular nucleus structures were strongly stained (Figures 1d, e). However, the intracellular site associated with fiber formation was impossible to be identified. Therefore, direct observation using DAPI was not suitable for visualization of intracellular structures at the fiber elongation.

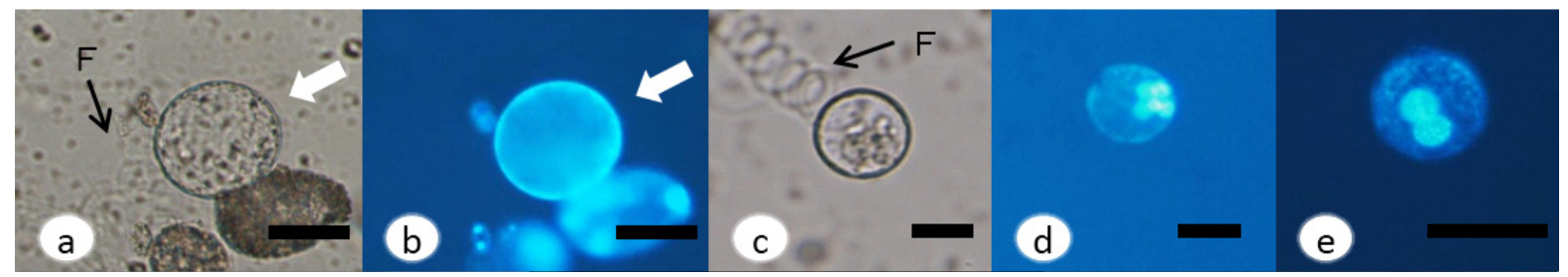

Figure 1. Photographs of fiber-forming Larix protoplasts under a fluorescence inverted microscope. After staining with DAPI, in bright-field (a, c) and under UV (b, d, e); a, b, c: without fixation; d, e: after fixation with glutaraldehyde; White arrow: a fiber forming protoplast; F: a fiber. Bar $=50 \mu \mathrm{m}$

\subsubsection{Observation by LCSM}

Phalloidin, an agent that binds to actin protein, is used for visualization of the cytoskeleton for cellulose ( $\beta$-1,4-glucan) microfibril deposition (Wightman \& Turner, 2008). Actin may play an important role in callose fiber formation, though the $\beta$-1,3-glucan component, and the sizes of fibril and sub-fibril structures (Fukumoto et al., 2005) are different from those of cellulose microfibrils. However, phalloidin can not penetrate cells readily. In order to improve the visualization of intracellular site associated with callose fiber formation, we devised a protocol using electric treatment to enhance the penetration of phalloidin into cells. As shown in Table 1, the viability of protoplasts was reduced at the highest electric treatment, $4 \mathrm{kV} / \mathrm{cm}$. We performed electric treatment at $3 \mathrm{kV} / \mathrm{cm}$, when the viability was more than $80 \%$ of the control, in the protoplast culture medium containing phalloidin. $2 \mathrm{kV} / \mathrm{cm}$ was selected for electric cell fusion of broad leaved trees in the medium containing only 2.5 
$\mathrm{mM}$ of $\mathrm{CaCl}_{2}$ for our previous study (Wakita et al., 2005). The density of the protoplasts used in this paper for electric treatment was $1 / 25$ of that for cell fusion. The salt concentration and cell density may have affected the electric conductance and the viability of protoplasts.

Table 1. Effects of voltage for DC pulse treatment on the viability of Larix protoplasts

\begin{tabular}{cc}
\hline $\mathrm{DC}(\mathrm{kV} / \mathrm{cm})$ & Viability $(\% \pm$ S.E. $)$ \\
\hline 0 & $83.8 \pm 1.4$ \\
2 & $77.4 \pm 1.3$ \\
3 & $72.3 \pm 4.5$ \\
4 & $56.4 \pm 4.0$ \\
\hline
\end{tabular}

As shown in the Figure 2, the fiber-forming protoplasts of Larix were clearly stained with Alexafluor 488 phalloidin. Phalloidin was localized to two different sites within the protoplast; a larger site from which fibers were absent, and a smaller site from which fibers were present. Therefore the actin protein may play a role in callose fiber formation at intracellular site. The background fluorescence was reduced greatly by purification of fiber-forming protoplasts using a micromanipulator. A manual type micromanipulator (MM-89) was sufficient for efficient transfer of protoplasts.

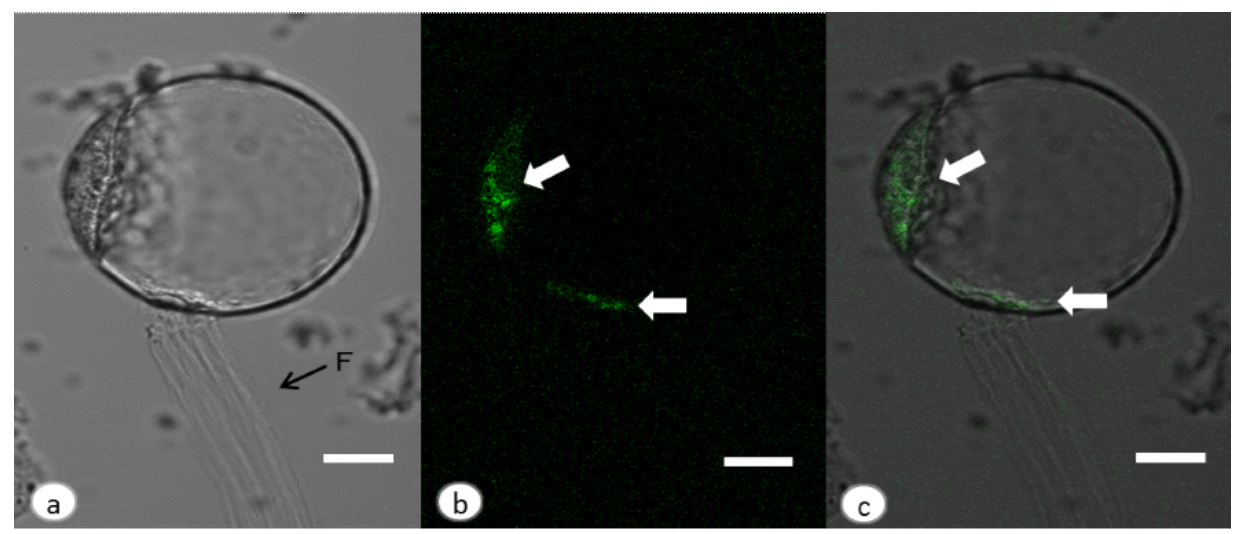

Figure 2. LCSM micrographs after staining with Alexafluor 488 phalloidin after electric treatment. a: DIC; b: fluorescence at $488 \mathrm{~nm}$; c: merge. F: a fiber. White arrows: fluorescent sites. Bar $=20 \mu \mathrm{m}$

As shown in Figures 1c-e, fiber-forming protoplasts containing a large nucleus were visualized by staining with DAPI under a fluorescence inverted microscope. These data are consistent with our previous finding, in which the specific site of fiber elongation was different from the large black site corresponding to the nucleus under an inverted microscope (Sasamoto et al., 2003).

LCSM-visualization of actin cytoskeletal dynamics in living cells of tobacco BY-2 cells was developed by transformation of the fusion gene of green fluorescent protein (GFP)-actin binding protein (Sano et al., 2005). However, such a method is not practical because of the low efficiency of callose fiber formation in a protoplast culture of tobacco BY-2 cells with a high concentration of salts (Fukumoto et al., 2005; Fukumoto, 2006). We tried introducing the GFP-gene into Larix protoplasts by electroporation using strong promoter genes, but direct observation of callose fiber formation failed in the cells expressing the GFP genes (Kurita et al., 2009).

\subsection{Observation of the Site of Fibril Formation}

\subsubsection{Observation by DIC}

Figure 3a shows the DIC observation of the site of fiber elongation from a Larix protoplast. Some surface structure could be observed. This site might be similar to the protruding surface structure of a fiber-forming protoplast under an inverted microscope (Sasamoto et al., 2003). 

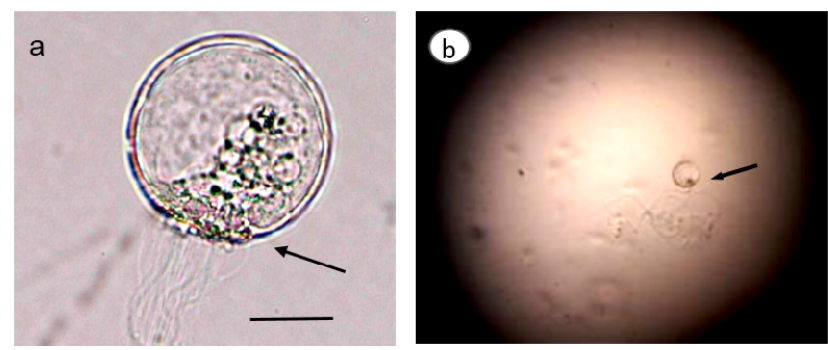

Figure 3. Photographs of the site of fiber formation at the surface of a Larix protoplast. a: DIC, Bar $=20 \mu \mathrm{m}$. b: in an No. 7 agarose bead with a low gelling temperature under an inverted microscope

\subsubsection{Observation by Single-Cell TEM}

Single-cell TEM was applied for observation of a protoplast by using an automatic micromanipulation system and ultra-low gelling temperature agarose No. 9 (Faure et al., 1992). As shown in Figure 3b, an elongated fiber forming protoplast of Larix was transferred efficiently to an agarose No. 7 bead with a diameter of $c a .3 \mathrm{~mm}$ using a manual type micromanipulator. Unlike ultra-low gelling temperature agarose No. 9, which easily liquidified, No. 7 agarose was solidified at room temperature and better to be handled.

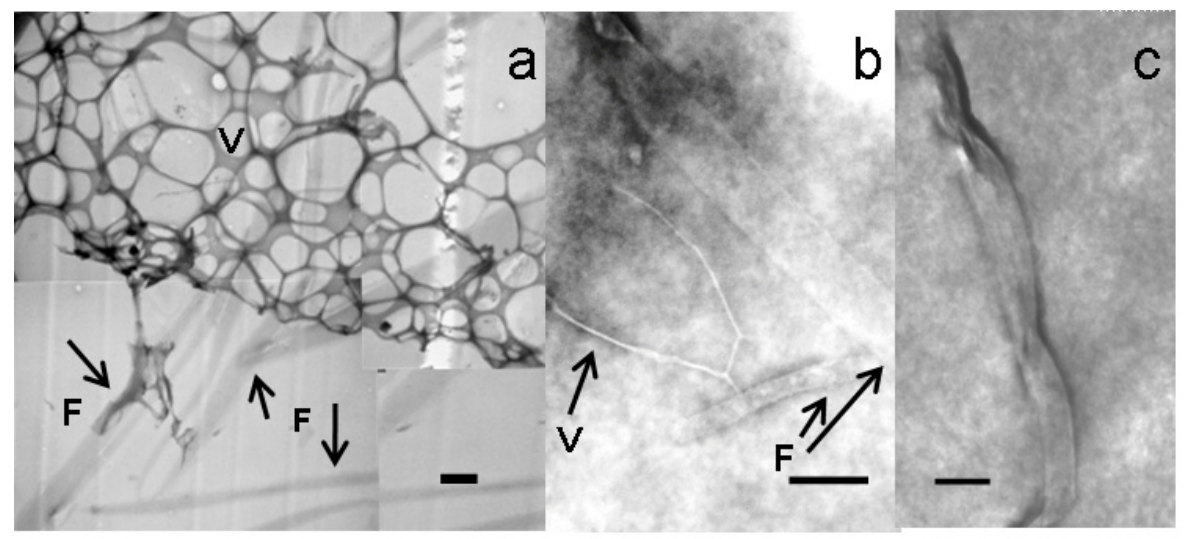

Figure 4. Single-cell TEM micrographs ( $a, b, c)$ at the site of (sub)-fibril formation of Larix protoplasts.

F: (sub)-fibril structure; V: vesicle-like structure. $B a r=2 \mu \mathrm{m}$

In TEM observation, small fibril structures were observed near the small vesicle-like structure (Figures $4 \mathrm{a}, \mathrm{b}$ ). The site might correspond to the surface sturucture of a protoplast in Figure 3a. These fibril structures had a size similar to the reported diameters of fibril and sub-fibril of Larix protoplast fiber, i.e., $0.7 \mu \mathrm{m}$ and $0.17 \mu \mathrm{m}$, respectively, calculated by LCSM and atomic force microscopy with image J analysis (Fukumoto et al., 2005; Kurita et al., 2009). Vesicle-like structures might be the exact site to form (sub)-fibrils of a protoplast fiber. The amount of callose at the vesicle-like site is considered to be low, because the fluorescent dye, Aniline Blue, stains only large elongated fibers but not the surface of a protoplast (Fukumoto et al., 2005). The mechanism for the formation of large spiral elongated fibers with a diameter of 10-20 $\mu \mathrm{m}$ remains to be clarified. Large spiral elongated fibers may be formed by the twisting force of the fibrils caused by the molecular structure of $\beta-1,3$-bond of callose. Figure $4 \mathrm{c}$ shows a twisted structure of a fibril. Therefore, single-cell TEM is a powerful tool to observe sub-fibril and fibril structures.

We are studying the mechanical properties of the novel callose fibers (Kurita et al., 2009; Oyanagi et al., 2012). A subfibril-fibril-large spiral fiber structures may affect the mechanical properties of them. Such a work might be useful for development of an alkali-labile nanomachine at a cellular level. 


\section{Conclusion}

The electric treatment improves the staining of protoplasts forming callose fibers using the non-permeable fluorescent dye, Alexafluor 488 phalloidin. The specific actin-binding intracellular site of Larix protoplasts was observed under LCSM. It was located at the membrane of callose fiber elongation and was a different site from the nucleus. (Sub)-fibril structures could be observed by single-cell TEM near the small vesicle-like structures.

\section{References}

Faure, J. -E., Mogensen, H. L., Kranz, E., Digonnet, C., \& Dumas, C. (1992). Ultrastructural characterization and three-dimensional reconstruction of isolated maize (Zea mays L.) egg cell protoplasts. Protoplasma, 171, 97-103. http://dx.doi.org/10.1007/BF01403723

Fukumoto, T. (2006). Analysis of novel callose fiber structures and control of their development in plant protoplast cultures. Ph.D. thesis of Yokohama National University. p. 115.

Fukumoto, T., Hayashi, N., \& Sasamoto, H. (2005). Atomic force microscopy and laser confocal scanning microscopy analysis of callose fibers developed from protoplasts of embryogenic cells of a conifer. Planta, 223, 40-45. http://dx.doi.org/10.1007/s00425-005-0065-3

Hayashi, N., Sugiyama, J., Okano, T., Ishihara, M., \& Shimizu, K. (1997a). Selective degradation of cellulose Ia component in Cladophora cellulose with Trichoderma viride cellulase. Carbohydrate Research, 305, 109-116. http://dx.doi.org/10.1016/S0008-6215(97)00281-4

Hayashi, N., Sugiyama, J., Okano, T., Ishihara, M., \& Shimizu, K. (1997b). The enzymatic susceptibility of cellulose microfibrils of the algal-bacterial type and the cotton-ramie type. Carbohydrate Research, 305, 261-269. http://dx.doi.org/10.1016/S0008-6215(97)10032-5

Kurita, A., Fukumoto, T., Hayashi, N., Shiraishi, T., \& Sasamoto, H. (2009). Characteristics of novel elongated callose fiber and regulatory mechanisms of the fiber formation in tree protoplast cultures: Mechanical properties; AFM; LCSM; TEM; GFP gene transformation. Proceedings of the 59th Annual Meeting of the Japan Wood Research Society, p. 96.

Kurita, A., Hasegawa, A., Hayashi, N., \& Sasamoto, H. (2008). Novel Callose fiber formation developed in protoplast cultures of a mangrove plant. Proceedings of the 72th Annual Meeting of the Botanical Society of Japan, p. 233.

Larkin, P. J. (1976). Purification and viability determination of plant protoplasts. Planta, 128, 213-216. http://dx.doi.org/10.1007/BF00393231

Murashige, T., \& Skoog, F. (1962) A revised medium for rapid growth and bioassay with tobacco tissue cultures. Physiologia Plantarum, 15, 473-497. http://dx.doi.org/10.1111/j.1399-3054.1962.tb08052.x

Ogita, S., Sasamoto, H., \& Kubo, T. (1999). Selection and microculture of single embryogenic cell clusters in Japanese conifers: Picea jezoensis, Larix leptolepis and Cryptomeria japonica. In Vitro Cellular and Developmental Biology Plant, 35, 428-431. http://dx.doi.org/10.1007/s11627-999-0061-6

Oyanagi, T., Hasegawa, A., Sasamoto, H., Hayashi, N., \& Fukumoto, T. (2012). Cell divisions and callose fiber formation in cultures of protoplasts isolated from suspension cells of a mangrove plant, Sonneratia caseolaris. Proceedings of the 62nd Annual Meeting of the Japan Wood Research Society, p. 116.

Sano, T., Higaki, T., Oda, Y., Hayashi, T., \& Hasezawa, S. (2005). Appearance of actin micfofilament 'twin peaks' in mitosis and their function in cell plate formation, as visualized in tobacco BY-2 cells expressing GFP-fimbrin. Plant Journal, 44, 595-605. http://dx.doi.org/10.1111/j.1365-313X.2005.02558.x

Sasamoto, H., Ogita, S., Hayashi, N., Wakita, Y., Yokota, S., \& Yoshizawa, N. (2003). Development of novel elongated fiber-structure in protoplast cultures of Betula platyphylla and Larix leptolepis. In Vitro Cellular and Developmental Biology Plant, 39, 223-228. http://dx.doi.org/10.1079/IVP2002388

Sasamoto, H., Wakita, Y., Yokota, S., \& Yoshizawa, N. (2000). Large electro-fused protoplasts of Populus alba selected by a micromanipulator: Techniques and some characteristics of cells and their regenerants. Journal of Forest Research, 5, 265-270. http://dx.doi.org/10.1007/BF02767120

Wakita, Y., Yokota, S., Yoshizawa, N., Katsuki, T., Nishiyama, Y., Yokoyama, T., ... Sasamoto, H. (2005). Interfamilial cell fusion among leaf protoplasts of Populus alba, Betula platyphylla and Alnus firma: assessment of electric treatment and in vitro culture conditions. Plant Cell, Tissue and Organ Culture, 83, 319-326. http://dx.doi.org/10.1007/s11240-005-8428-9 
Wightman, R., \& Turner, S. R. (2008). The role of the cytoskeleton during cellulose deposition at the secondary cell wall. Plant Journal, 54, 794-805. http://dx.doi.org/10.1111/j.1365-313X.2008.03444.x

\section{Copyrights}

Copyright for this article is retained by the author(s), with first publication rights granted to the journal.

This is an open-access article distributed under the terms and conditions of the Creative Commons Attribution license (http://creativecommons.org/licenses/by/3.0/). 